\title{
Validation of Numerical Modeling for Flows near Draghead in Operation
}

\author{
by Morgan M. Johnston, Allen Hammack, and David L. Smith
}

BACKGROUND: Fish and other aquatic species occasionally become entrained in dredges during operation. To better predict the likelihood of this entrainment occurring, the U.S. Army Corps of Engineers, San Francisco District, contacted the U.S. Army Engineer Research and Development Center (ERDC) to conduct a numerical model study to determine the flow behavior surrounding a draghead during operation. The validation data were taken from a previously completed 1:7-scale physical model study of a California draghead. ${ }^{1}$ The numerical model study was completed by validating the numerical modeling process and then performing simulations at prototype scale to provide appropriate velocity measurements in the field. This report focuses on the numerical modeling process and validation portion of the numerical model.

NUMERICAL CODE: The three-dimension (3D) Reynolds-Averaged, Navier-Stokes (RANS) module of Adaptive Hydraulics (AdH) was selected for the numerical model effort. The RANS equations for incompressible flows are the conservation of mass as

$$
\nabla \cdot \boldsymbol{U}=0
$$

and the conservation of momentum as

$$
\rho\left(\frac{\partial U}{\partial t}+\boldsymbol{U} \cdot \nabla \boldsymbol{U}\right)-\nabla \cdot \sigma+\nabla \cdot(\rho \boldsymbol{u u})=0
$$

where:

$$
\begin{aligned}
\rho & =\text { fluid density } \\
t & =\text { time } \\
\boldsymbol{U} & =\text { total velocity vector at a location in the flow field } \\
\boldsymbol{U} & =\overline{\boldsymbol{U}}+\boldsymbol{u}^{2} \\
\sigma & =-p \boldsymbol{I}+\tau^{3} \\
p & =\text { thermodynamic pressure } \\
\boldsymbol{I} & =\text { identity matrix } \\
\mathrm{T} & =2 \mu \boldsymbol{\Gamma} \\
\boldsymbol{\Gamma} & =0.5 \cdot\left(\nabla \boldsymbol{u}+\nabla \boldsymbol{u}^{T}\right)^{4} \\
\mu & =\text { fluid viscosity. }
\end{aligned}
$$

\footnotetext{
${ }^{1}$ Figure 2 in Bryant, D. B., B. C. McFall, R. E. Randall, Y. Zhi, T. L. Welp, and D. L. Smith. In preparation. Physically Modeling of Flow Field around a Draghead. ERDC/CHL Technical Report. Vicksburg, MS: U.S. Army Engineer Research and Development Center. U.S. Army Engineer Research and Development Center.

${ }^{2}$ Where $\overline{\boldsymbol{U}}$ is the time-average velocity and $\boldsymbol{u}$ is the fluctuating velocity.

${ }^{3} \sigma$ is the fluid viscosity including viscosity induced due to flow characteristics.

${ }^{4}$ Where ${ }^{T}$ is the matrix transpose.
} 
RANS-AdH is referred to as a non-hydrostatic numerical flow model because it makes no assumptions regarding pressure distribution. Accurately calculating the turbulent flow parameters is critical to the accuracy of the flow solution for high Reynolds number flows. While Equation 2 uses shear velocity to model turbulent flow, turbulent flow can also be modeled using a constant eddy viscosity. Currently, RANS-AdH captures the turbulent flow dissipation via a constant eddy viscosity subgrid closure scheme included in the molecular viscosity portion of the momentum equations. RANS-AdH has an automatic mesh adaption capability that is used to ensure a mesh independent flow solution (Tate, Berger, and Stockstill 2006; Savant et al. 2018). Mesh adaption in RANS-AdH is controlled using the same method as in two-dimensional shallow water AdH by two user-specified parameters: a mesh refinement tolerance and a maximum number of adaption levels (ERDC 2019). The "error" from different flow information is collected, and a single residual value is formed. This residual is used to determine an appropriate refinement tolerance for the simulation. RANS-AdH refines the mesh when the calculated residual is above the set refinement tolerance. The amount of refinement allowed depends on the levels of adaption specified. Further information regarding general mesh adaption can be found in Oden et al. (1990) and Thompson, Warsi, and Mastin (1985).

GEOMETRY DESCRIPTION: The facility used in the physical model experiments utilized a 45.7-meter (m) long, $3.7 \mathrm{~m}$ wide, and $1.7 \mathrm{~m}$ deep tank. Figure 1 shows schematics of the physical testing facility. These dimensions were used to generate the flow domain for the numerical model.

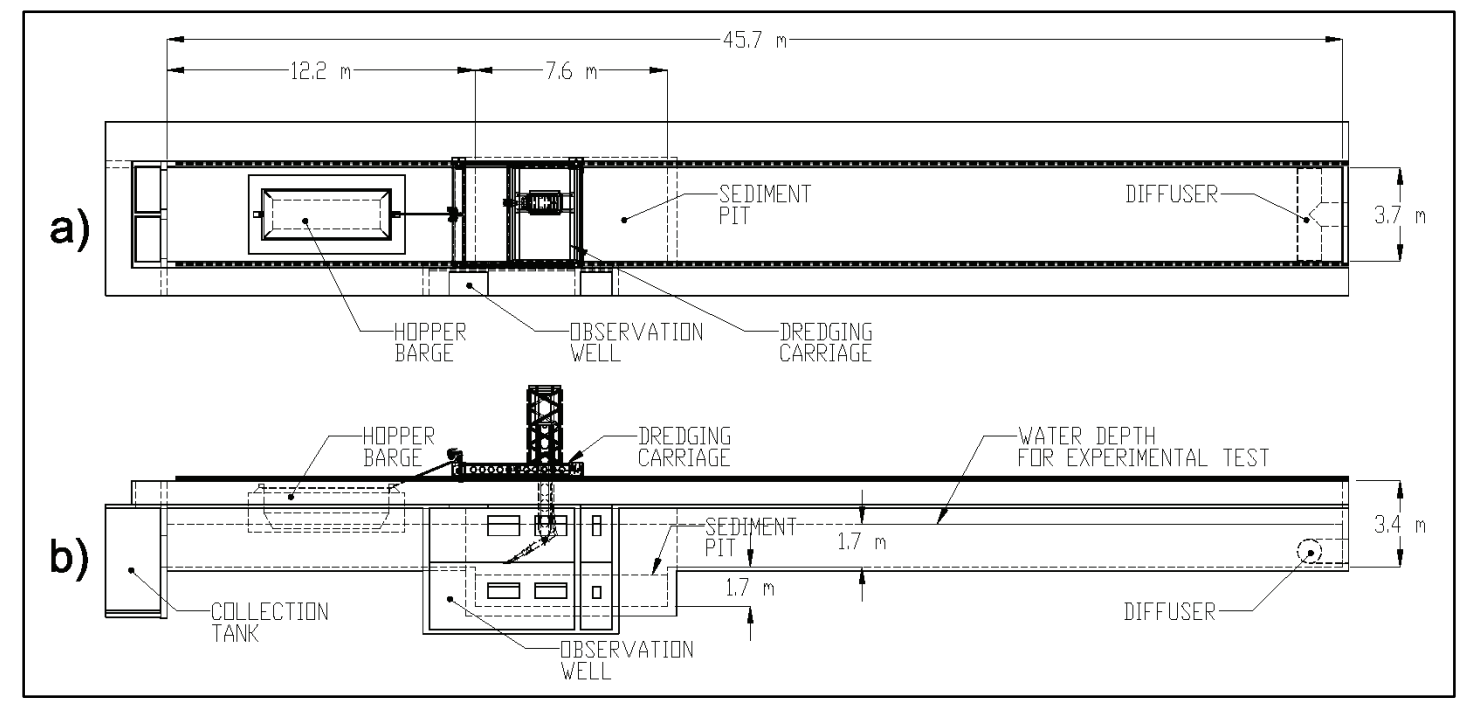

Figure 1. Schematic drawings of the physical testing facility ${ }^{1}$.

The 3D computer aided drafting (CAD) drawing of the 1:7-scale California draghead (shown in Figure 2) was used to create the draghead for the numerical model. The original CAD drawing is appropriate for the creation of the physical model, but manipulation is necessary for sufficiently high-quality 3D meshes to be generated. Such modifications are common for a numerical model study because details of the drawing that are either necessary or inconsequential for a physical

${ }^{1}$ Figure 2 in Bryant, D. B., B. C. McFall, R. E. Randall, Y. Zhi, T. L. Welp, and D. L. Smith. In preparation. Physically Modeling of Flow Field around a Draghead. ERDC/CHL Technical Report. Vicksburg, MS: U.S. Army Engineer Research and Development Center. 
model (such as small details like nuts and bolts for connections or small lips for flanges) commonly cause difficulties in generating computational meshes and increase computational burden.

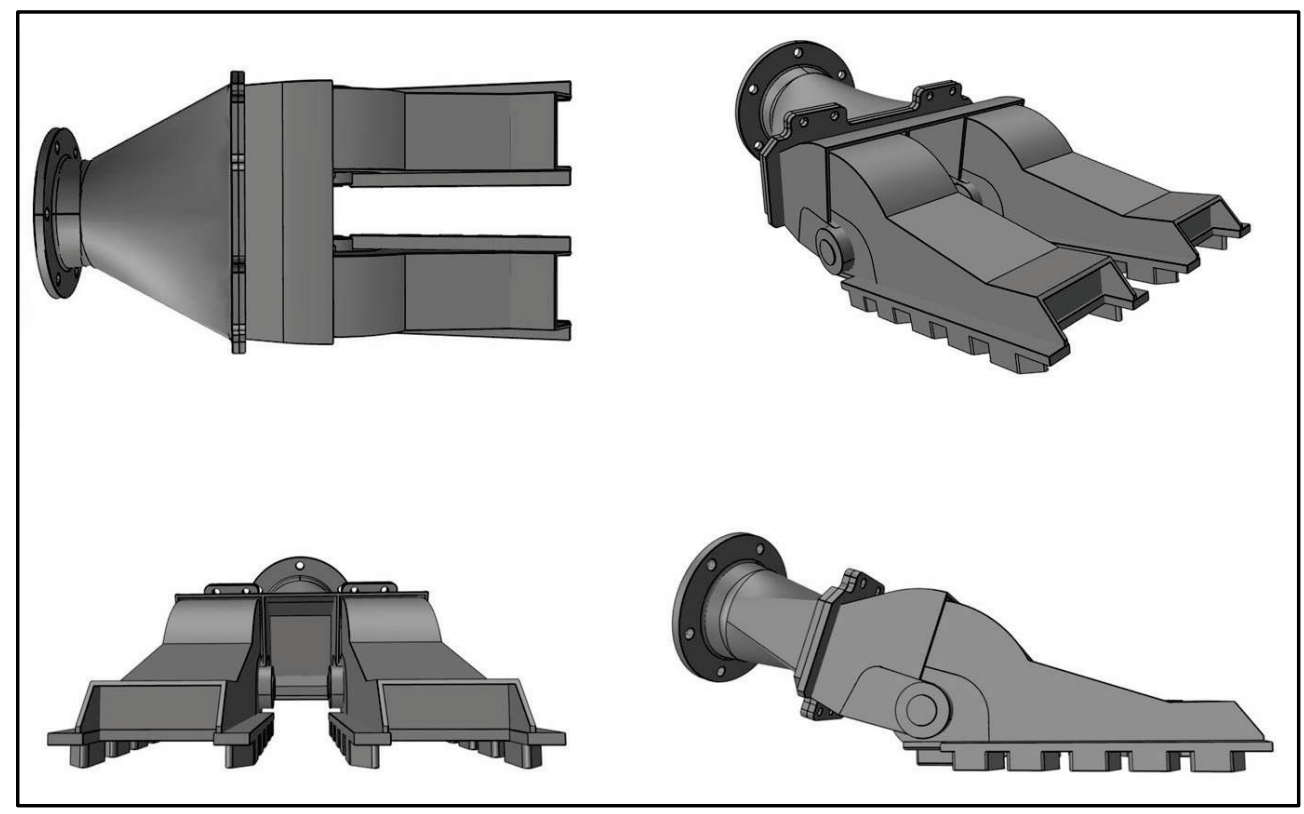

Figure 2. Various views of original 3D CAD drawing.

A geometry modification is justifiable if it causes a negligible change to the flow solution in the area of interest but either reduces the number of nodes and elements in the mesh or significantly improves the mesh quality. For the draghead geometry, extraneous edges were eliminated, and non-vital geometry components were simplified. Previous modeling experience has indicated that both types of these modifications could eliminate extra refinement for little or no flow solution change. Examples of these changes are shown in the circled areas in Figure 3. The top image shows the original geometry, and the bottom image shows the modified geometry. The areas in the red circles show surfaces that were made flush with the surrounding geometry to eliminate unnecessary resolution in those areas. The green circle highlights an area where extraneous curves were removed to simplify the geometry. Areas in the blue circles show holes that were filled in the geometry to reduce mesh resolution and to eliminate areas that are known to cause difficult flow conditions, yet are insignificant to the flow solution area of interest.

Modifying the geometry to maintain a good quality and reasonably sized mesh usually comprises most of the 3D modeling effort. Many necessary modifications can be determined from previous 3D numerical modeling experience, but often problems do not become apparent until some simulations have been completed and the solutions have been analyzed. When additional modifications are discovered, the geometry must be revised and re-meshed. Boundary conditions must be reapplied and initial simulations must be re-submitted. Then, simulation solutions can be analyzed to determine if the modification produced the desired effect. 


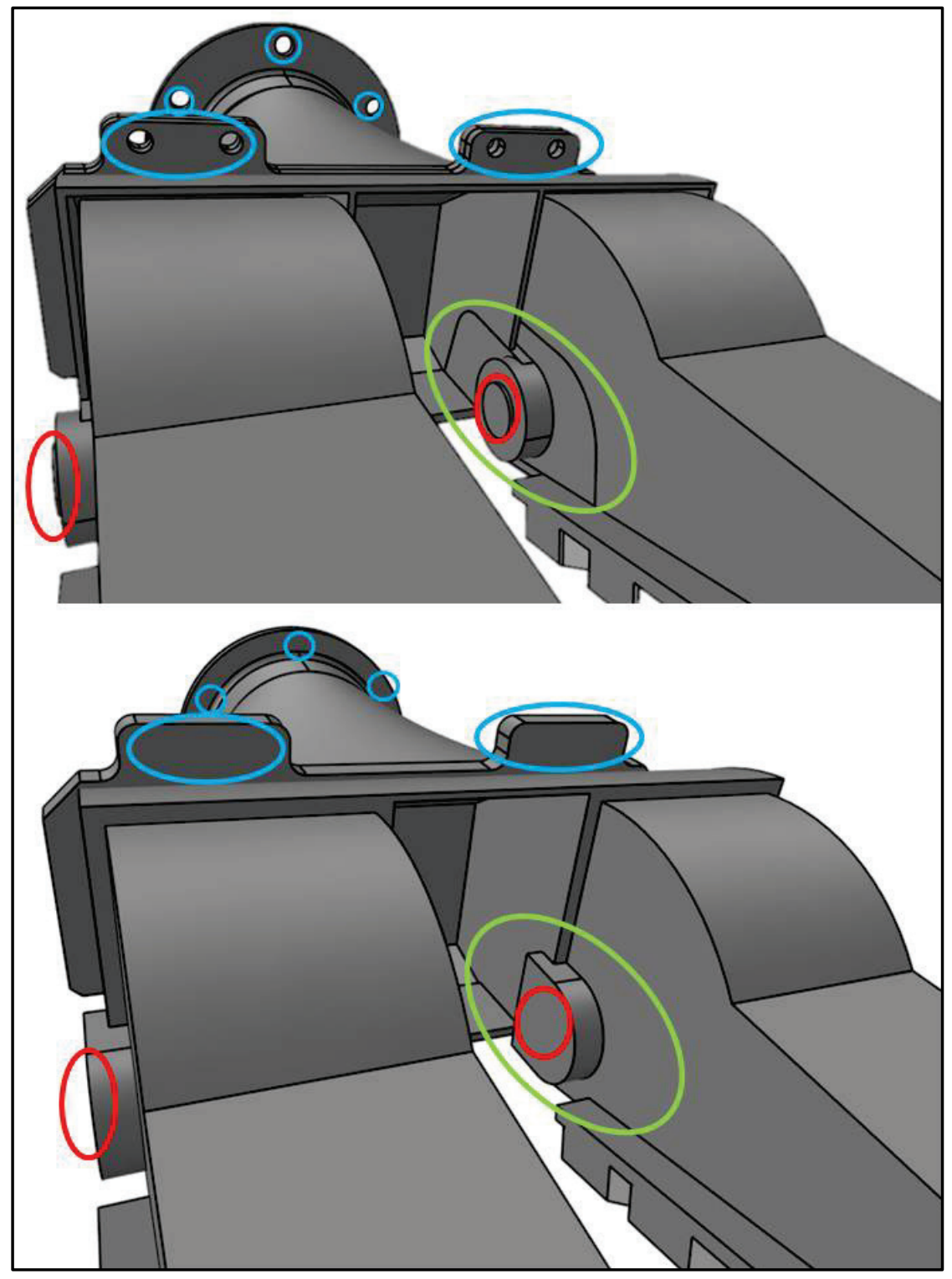

Figure 3. Geometry modifications for meshing purposes.

MESHING: Flow solution accuracy and reasonable simulation times require a high-quality mesh (mesh quality $\geq 0.3$ ). While RANS-AdH has an automatic mesh adaption option available, it does not transform a low-quality mesh into a high-quality mesh. A higher-resolution mesh may generate a higher-quality mesh, but it will also require larger processing demands and might require longer processing time. Therefore, the element count must be limited when possible. However, if high resolution is required to appropriately represent important geometry, then element count cannot be reduced in those areas.

Areas with bad quality elements should also be considered during geometry modification. The user must decide whether a reasonable amount of extra resolution would increase the quality enough to remove the area of concern or if a geometry modification might be more appropriate. For the draghead, extra resolution was added to fillets to obtain a more accurate representation of the geometry as the overall increase to node count was small. 
Mesh information for the final model scale mesh is shown in Table 1. Due to the overall complexity and small size of the model scale draghead, small elements were required to adequately capture the intricate details of the draghead. The tank was much larger and less complex than the draghead, so it could be represented by much larger elements. Figure 4 shows varying levels of mesh resolution in the model scale mesh prior to adaption.

\begin{tabular}{|c|c|}
\hline Model scale mesh element count & $1,978,757$ \\
\hline Model scale mesh node count & 375,393 \\
\hline Approximate largest element length $\left(\mathrm{cm}^{*}\right)$ & 37 \\
\hline Approximate smallest element length $(\mathrm{cm})$ & 0.07 \\
\hline
\end{tabular}

${ }^{*} \mathrm{Cm}=$ centimeter

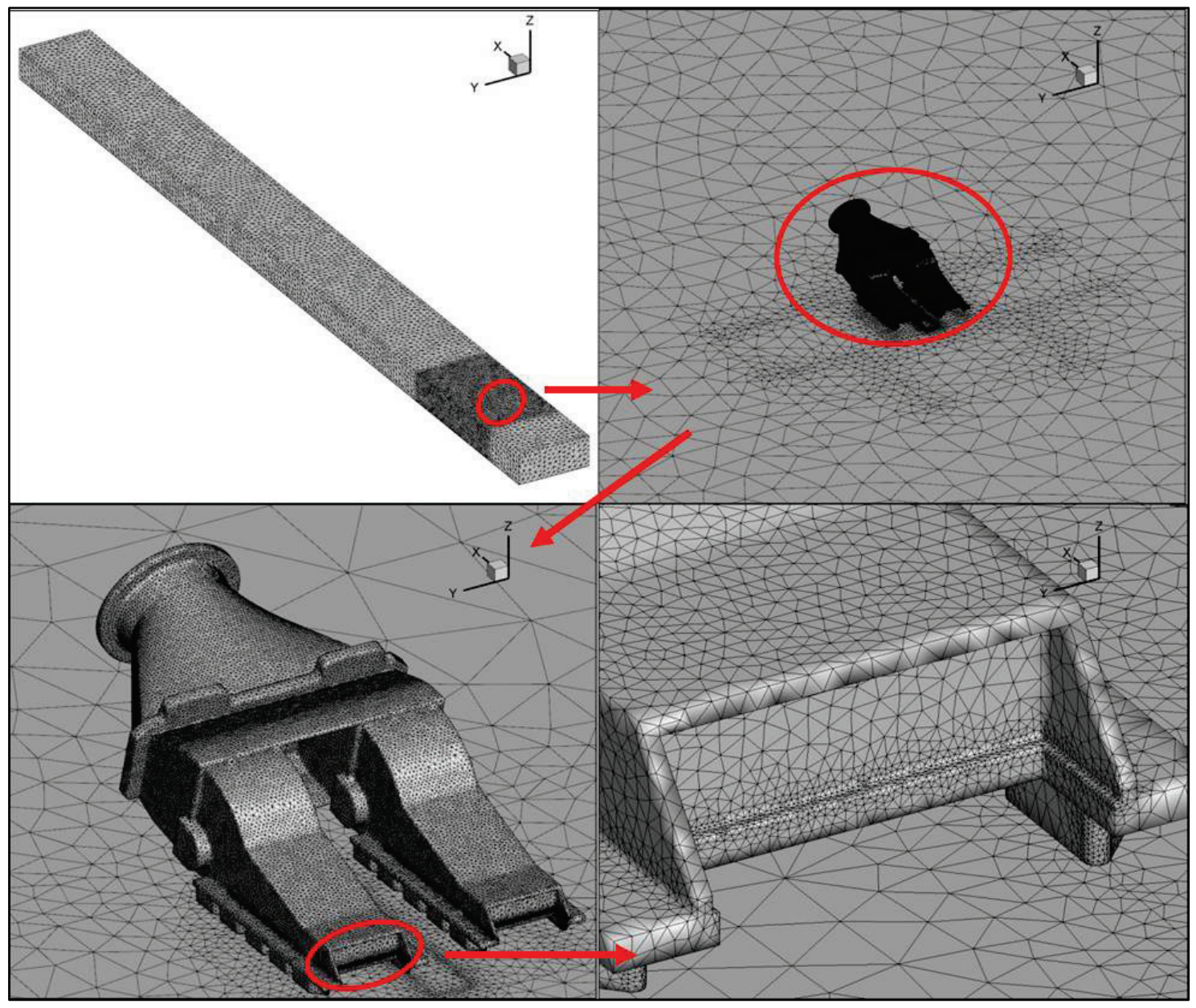

Figure 4. Varying levels of mesh resolution. 
BOUNDARY CONDITIONS: The flow condition selected to validate the numerical model was a stationary draghead located 2.5 centimeters above the concrete floor with a 15.7 liters per second (250 gallons per minute) discharge into the draghead. Surfaces were created along the bottom of the draghead so the directed flow could be specified into the draghead. The specified velocity was directed normal to these surfaces. Figure 5 shows the inflow surfaces in red. A uniform velocity was assumed across all inflow surfaces because there were no data to support an implementation of a different flow distribution. Therefore, the average velocity (total discharge divided by inflow area) was applied to each node on every inflow surface. A hydrostatic pressure boundary condition was assigned to the nodes located at the tank wall upstream of the draghead. This hydrostatic pressure designates zero pressure at a previously established water surface elevation (in this case, the depth of the tank). Since the draghead is positioned very close to the tank floor and the flow velocities away from the draghead are small, a frictionless fixed-lid boundary condition was used for the top of the tank. This boundary condition prevents movement of the water surface throughout the simulation. All other surfaces were set as no flux boundaries to prevent flow to pass through them. A Darcy friction factor of 0.0309 was applied to the draghead to represent plastic, and a Darcy friction factor 0.0644 was applied to the tank floor to represent concrete (Sturm 2001).

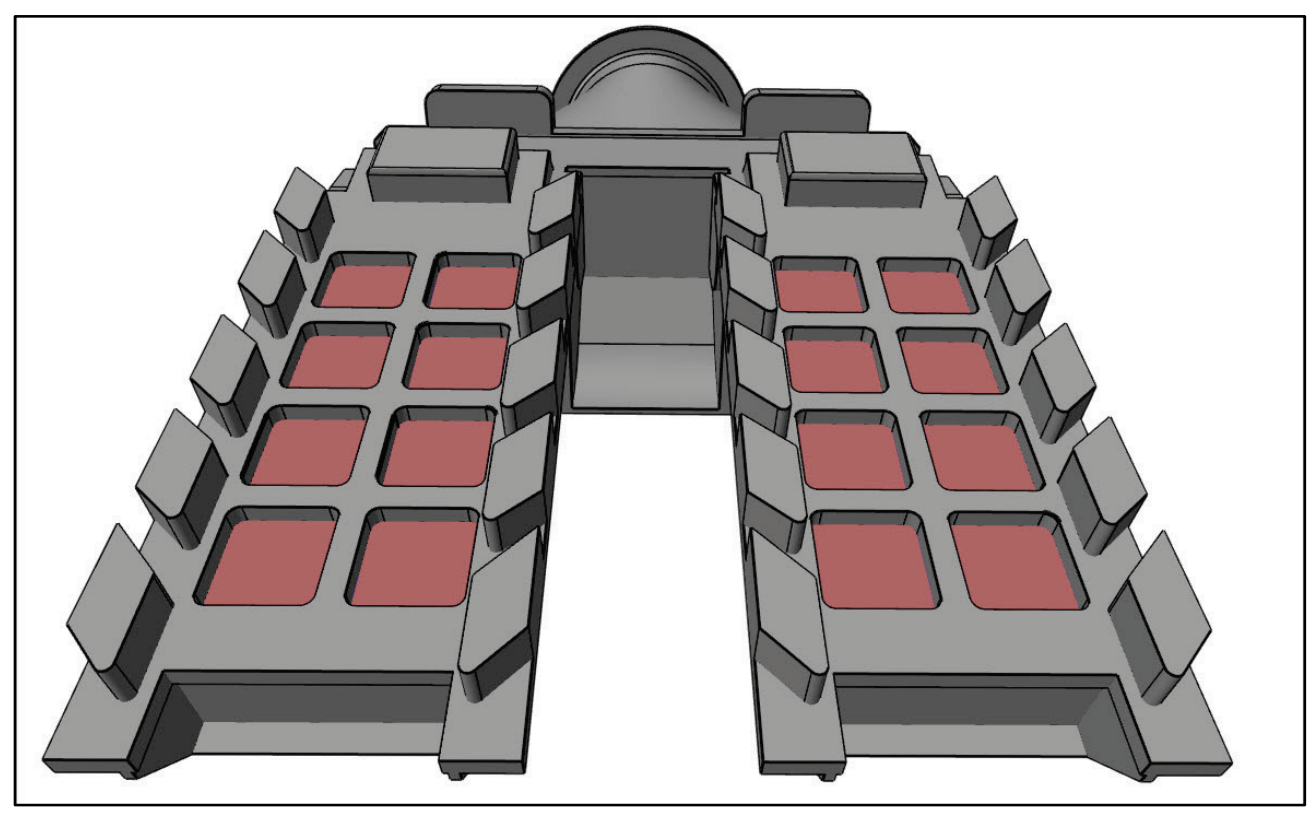

Figure 5. Created inflow boundaries are shown in red.

VALIDATION: The physical model provided mean velocity values for 16 reference points near the draghead. These reference points are shown in Figure 6. There are eight planwise locations (A$\mathrm{H})$ with two different elevation values at each location. The lower elevation point is labeled with a "1" while the higher elevation point is labeled with a "2." Each reference point corresponds to the coordinate of a node in the computational mesh, so the flow solution at each reference point was calculated directly by RANS-AdH. 


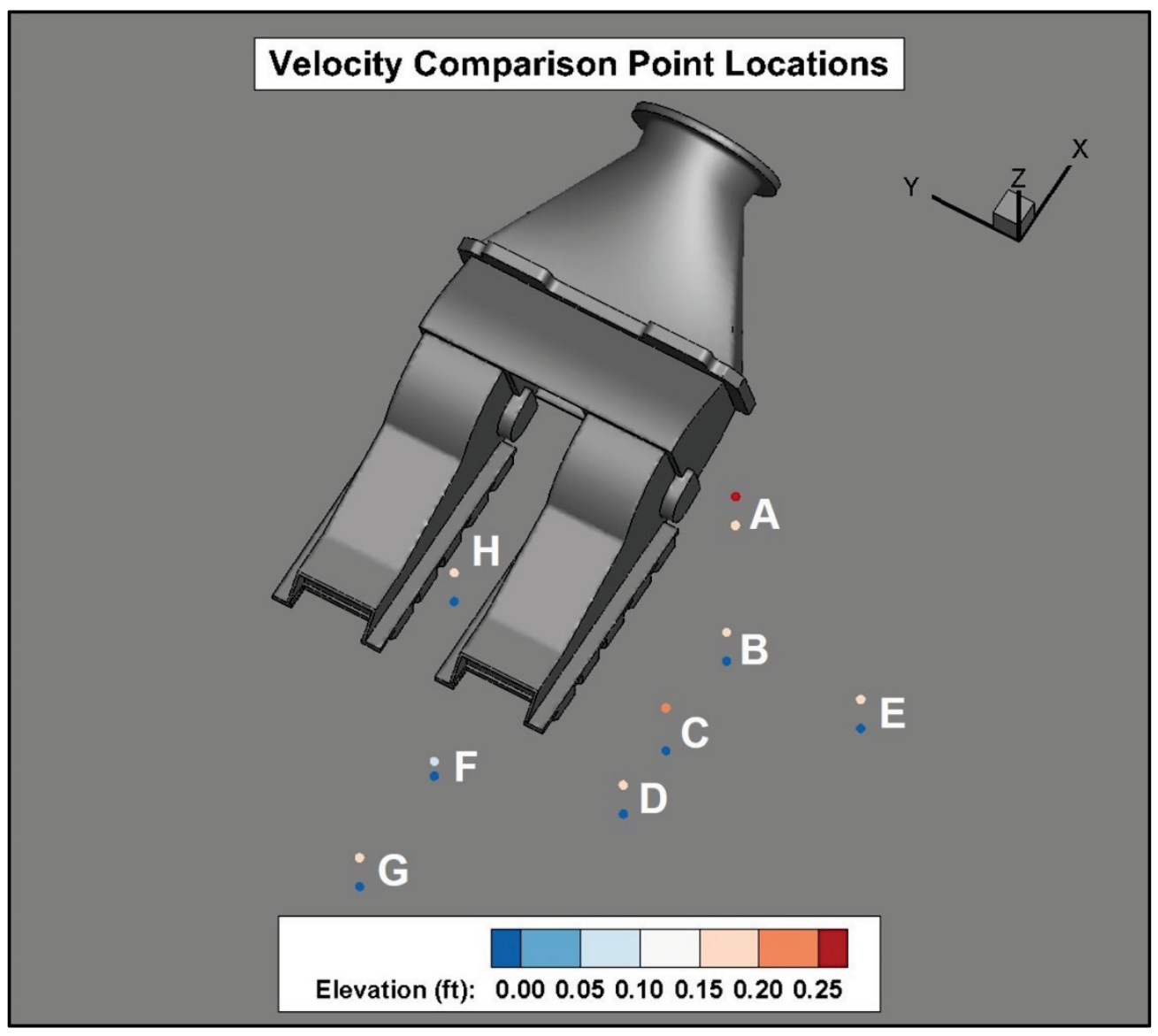

Figure 6. Locations of reference points.

In the initial simulation for each flow condition, a high eddy viscosity was set for the entire flow domain. A short simulation, typically 1 minute of simulated time, was then completed with this high initial eddy viscosity. Using the solution from the final time of the previous simulation, a new simulation with a reduced eddy viscosity was run. Once the new simulation was completed, the results were compared with the previous solution. If the solution was still changing, then a new simulation had to be submitted, which further reduced the eddy viscosity. This process continued until no flow solution change occurred. For the draghead, seven eddy viscosity iterations were required for the flow solution to converge.

The velocity values at each reference point in the numerical model maintained reasonable agreement with the physical model except for the two reference points located between the draghead toes (H1 and H2). Since, RANS-AdH uses a constant eddy viscosity to represent turbulence effects, a separate material between the draghead toes had to be created so the eddy viscosity in the area of those points could be modified independent of the rest of the flow domain. The geometry had to be modified and then re-meshed to add this necessary material.

Once the eddy viscosity was reduced for the entire flow domain, the eddy viscosity for the area between the draghead toes was slowly increased. The velocity values of the physical model were compared with the velocity values of the numerical model for the reference points $\mathrm{H} 1$ and $\mathrm{H} 2$ until an appropriate eddy viscosity for that area was determined. The fifth eddy viscosity simulated for the area between the toes was determined to be appropriate for the draghead simulation. Table 2 
shows the change in velocity magnitude caused by adding the extra material for eddy viscosity manipulation. While the velocity magnitude of some of the reference points shifted slightly away from the velocity magnitude of the paired physical model reference point, the largest change can be seen in $\mathrm{H} 1$ and $\mathrm{H} 2$. Both of these reference points experienced better agreement with the physical model from the addition of the extra material.

\begin{tabular}{||c|c|c|c||}
\hline \hline Table 2. Change in velocity magnitude due to eddy viscosity manipulation. \\
\hline \hline $\begin{array}{c}\text { Reference } \\
\text { Point }\end{array}$ & $\begin{array}{c}\text { Physical Model - } \\
\text { Velocity Magnitude } \\
\left(\mathrm{cm} / \mathrm{s}^{*}\right)\end{array}$ & $\begin{array}{c}\text { Numerical Model } \\
\text { Numerical Model (one } \\
\text { eddy viscosity defined) } \\
\text { Velocity Magnitude (cm/s) }\end{array}$ & $\begin{array}{c}\text { (additional eddy viscosity } \\
\text { defined) - } \\
\text { Velocity Magnitude (cm/s) }\end{array}$ \\
\hline A1 & 5.0 & 5.8 & 6.2 \\
\hline B1 & 5.9 & 6.4 & 7.1 \\
\hline C1 & 3.8 & 5.6 & 6.1 \\
\hline D1 & 2.9 & 4.0 & 4.2 \\
\hline E1 & 2.8 & 2.0 & 2.1 \\
\hline F1 & 3.8 & 6.7 & 7.2 \\
\hline G1 & 2.6 & 2.2 & 2.3 \\
\hline H1 & 20.0 & 29.4 & 17.4 \\
\hline A2 & 3.7 & 3.8 & 4.1 \\
\hline B2 & 4.9 & 4.9 & 5.4 \\
\hline C2 & 3.2 & 3.9 & 4.1 \\
\hline D2 & 3.2 & 3.5 & 3.6 \\
\hline E2 & 3.3 & 1.9 & 2.0 \\
\hline F2 & 3.9 & 6.1 & 6.5 \\
\hline G2 & 1.9 & 2.2 & 2.4 \\
\hline H2 & 11.2 & 18.7 & \\
\hline \hline
\end{tabular}

${ }^{*} \mathrm{~cm} / \mathrm{s}=$ centimeters per second

Once the eddy viscosity values were determined, levels of adaption and refinement tolerance were selected. For the draghead model, a velocity contour range encompassing a small diameter away from the draghead was used to select a refinement tolerance of 0.0005. Simulations were run with varying levels of adaption to reach a mesh independent solution. The 16 reference points were monitored at each level of adaption until the flow solution converged; this procedure is referred to as "mesh convergence" and provides confidence that numerical results are not biased due to spatial resolution of the mesh. Thus, the solution is ensured to be mesh independent. Figure 7 shows the incremental changes of velocity between the different levels of adaption for eight different reference points. Five levels of adaption were selected as the appropriate amount of adaption for these simulations. 


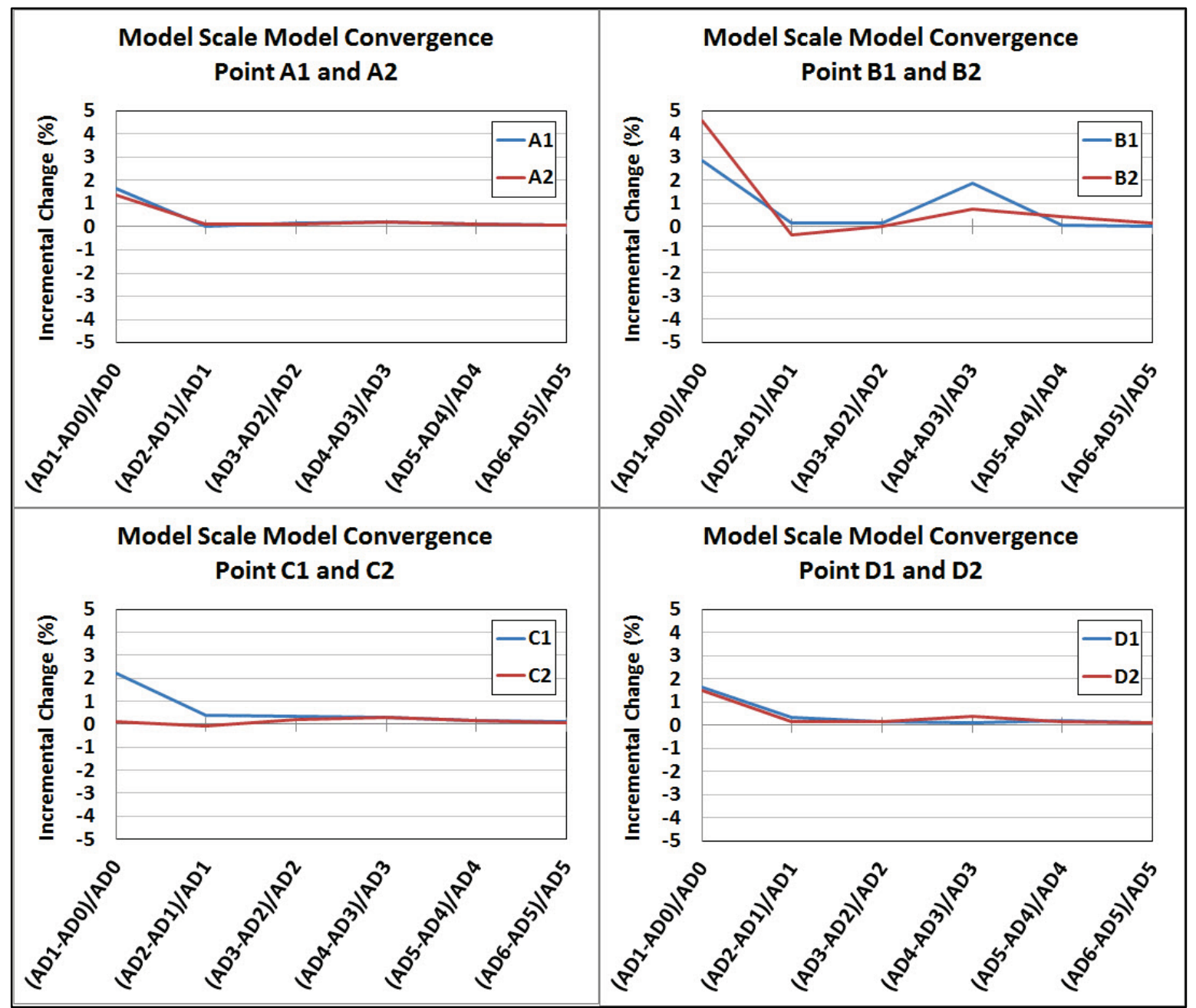

Figure 7. Solution convergence behavior for reference points A-D.

Figure 8 shows the number of nodes at each level of adaption. The increase of nodes for each adaption level increase is nonlinear. At the fourth level of adaption, the node increase becomes very large. Table 3 shows the node count and percent change for each level of adaption. Percent change overall was determined by subtracting the initial node count (prior to adaption) from the final node count of that level of adaption and then dividing by the initial node count. 


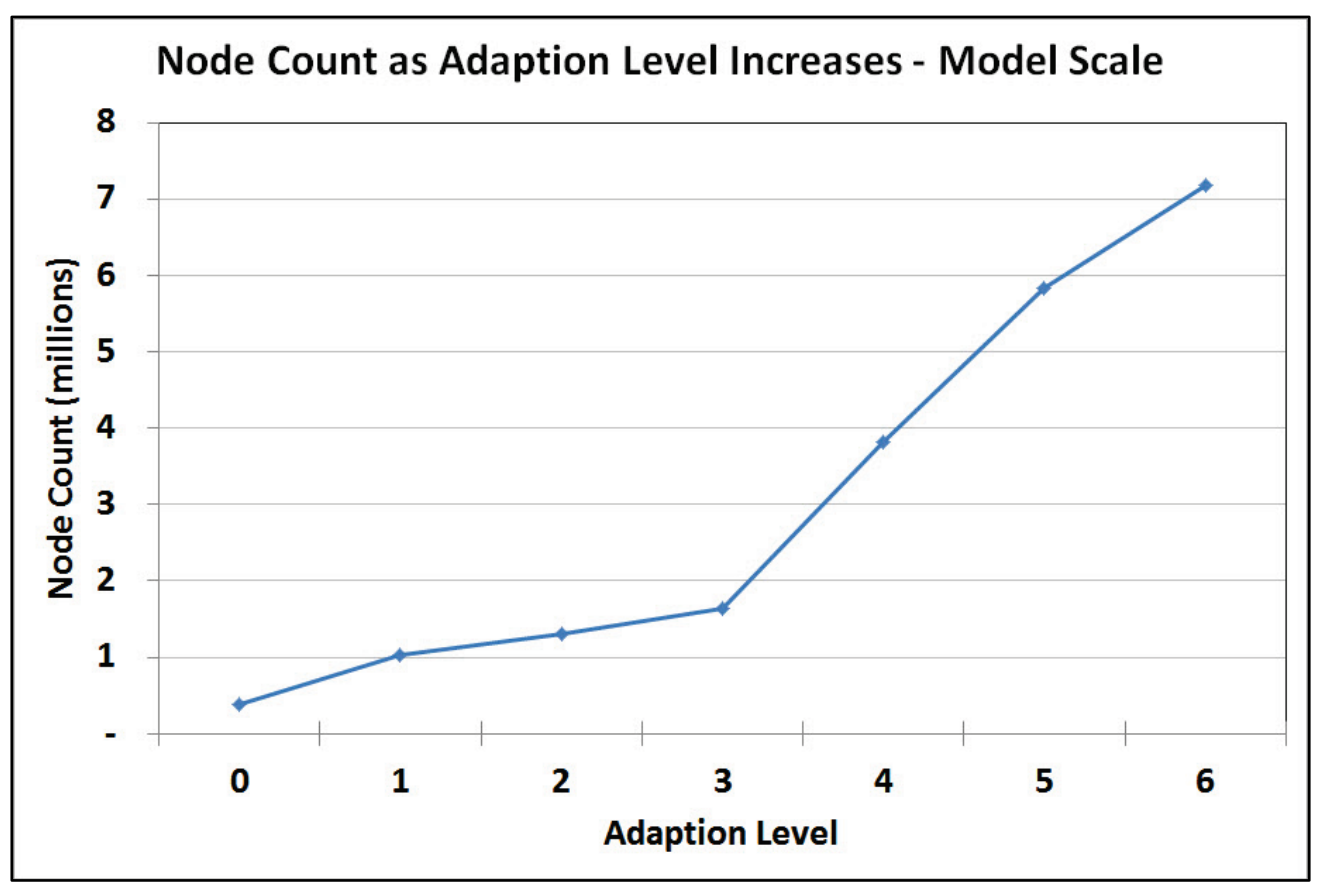

Figure 8. Node count as adaption level increase.

Table 3. Values for node count as adaption levels increase.

\begin{tabular}{|c|c|c|}
\hline Level of Adaption & Final Node Count & $\begin{array}{c}\text { Percent Change Overall } \\
(\%)\end{array}$ \\
\hline 0 & 375,393 & - \\
\hline 1 & $1,026,008$ & 173 \\
\hline 2 & $1,310,685$ & 249 \\
\hline 3 & $1,643,555$ & 338 \\
\hline 4 & $3,817,702$ & 917 \\
\hline 5 & $5,831,331$ & 1453 \\
\hline 6 & $7,184,225$ & 1814 \\
\hline
\end{tabular}

RESULTS: Figure 9 shows the velocity magnitude comparison between the physical and numerical model at the 16 reference points. The engineers who conducted the physical model study determined that $15 \%$ is a reasonable estimate of the uncertainty of the physical model results. Therefore, uncertainty bars of $15 \%$ have been added to the physical model velocity results in Figure 9 . The velocity magnitude results for 10 of the 16 reference points are within or very close to within $15 \%$ of the physical model measurements. Since such extensive efforts (discussed in the Validation section) were made to improve solution agreement, the authors expect that the agreement between numerical model and physical model results cannot be significantly improved without some capabilities added to RANS-AdH. The model validation results were reviewed by engineers in the Coastal and Hydraulics Laboratory (CHL), and the consensus is that the RANSAdH results sufficiently reproduce the physical model results. 


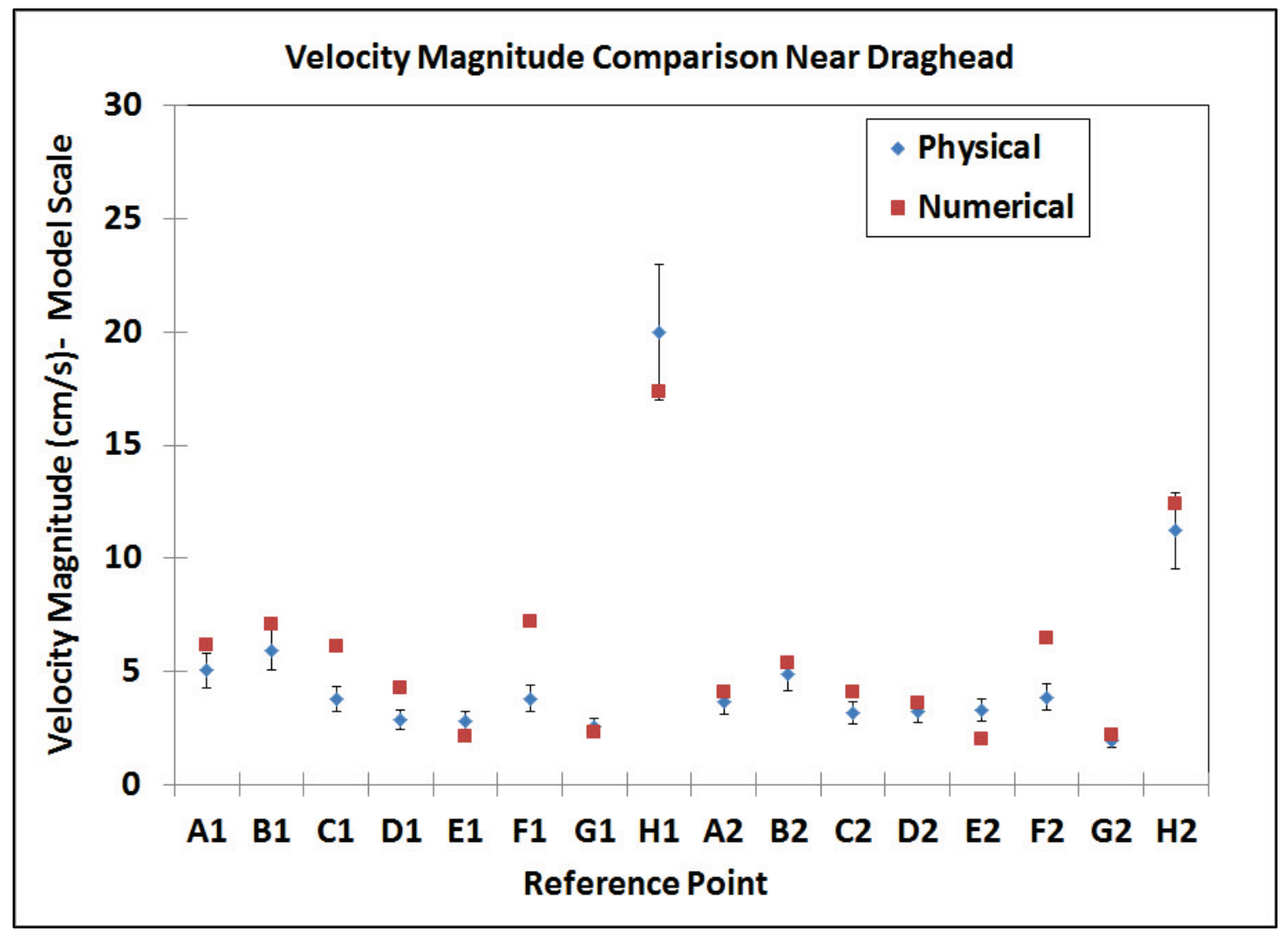

Figure 9. Velocity magnitude comparison for physical and numerical model.

CONCLUSION and FUTURE RESEARCH: The velocity magnitudes from the numerical model compare well with over half of the 16 reference points. For these simulations, it was deemed unnecessary to directly model the boundary layer in the mesh as the overall effects from the boundary layer would make a small impact on the general flow behavior while adding a large computational burden. Friction values were chosen based off the physical experiment parameters, but sensitivity on these selected friction values were not completed. The assumption of uniform flow across the inflow surfaces, not directly modeling the boundary layer, and not completing a sensitivity test on the friction values could account for some of the discrepancies between the numerical and physical model.

The flow situation is dominated by converging flow throughout the domain, with the one exception being the bifurcating flow present between the draghead toes. Converging flow has relatively low dissipation, with fewer eddies forming than with diverging flow, and a steady-state condition can be reached as long as the geometry is stationary, the boundary conditions are steady, and the flow velocities are relatively small. Regardless, accurate near-field flow remains difficult, so extreme care must be made when considering near-field flow results from a numerical model when physical model measurements are not available for validation.

The numerical modeling process and results may be improved if a k-epsilon (k-E) turbulence closure model is incorporated into RANS-AdH (Mellor and Yamada 1982; Bardina, Huang, and Coakley 1997). The current process for adjusting the turbulence parameters requires manually adjusting the eddy viscosity for different regions of the flow domain. These regions are created in the mesh, so if extra regions are determined necessary after the initial mesh is generated, then a 
new mesh and new boundary condition file must be created. This process is extremely laborintensive. The incorporation of a $\mathrm{k}-\varepsilon$ turbulence model would greatly reduce the total time necessary to complete a RANS-AdH simulation by removing the need of slowly dropping the eddy viscosity until the flow solution has converged and by eliminating the need to divide the flow domain into multiple regions solely to adjust the eddy viscosity when different eddy viscosity values are necessary in the same flow domain. The $\mathrm{k}-\mathcal{E}$ turbulence model would calculate the turbulent kinetic energy and turbulent dissipation rate at every node in the flow domain for every time step, so the turbulent flow behavior would be updated for each time step.

While the adaption option in RANS-AdH is an incredibly robust tool, an automatic mesh smoothing capability would be very beneficial. Currently, nodes are added to eliminate residuals higher than the refinement tolerance set; however, there is no element quality control while the simulation is running. The added resolution can and likely does reduce the quality of the mesh when many levels of adaption are employed during a simulation. An automatic mesh smoother would eliminate this concern, so a smoother should be applied once additional nodes are added to the mesh from adaption. This capability would reduce error while maintaining mesh quality.

By incorporating a two-equation turbulence closure model and a mesh smoother into RANS-AdH, the overall 3D simulation process could become streamlined. RANS-AdH simulations could reach more accurate results quicker by eliminating labor-intensive components of the current process and by improving the mesh adaption capability.

ADDITIONAL INFORMATION: For additional information, contact the Locks group in the Navigation Branch of ERDC, CHL, 3909 Halls Ferry Road, Vicksburg, MS 39180. Questions may be addressed to the following:

Morgan Johnston: 601-634-2365; email: Morgan.M.Johnston@erdc.dren.mil

Allen Hammack: 601-634-3628; email: Allen.Hammack@erdc.dren.mil

\section{REFERENCES}

Bardina, J. E., P. G. Huang, and T. J. Coakley. 1997. Turbulence Modeling Validation, Testing, and Development. NASA Technical Memorandum 110446. Moffett Field, CA: National Aeronautics and Space Administration, Ames Research Center.

Mellor, G. L., and T. Yamada. 1982. "Development of a Turbulence Closure Model for Geophysical Fluid Problems." Reviews of Geophysics and Space Physics 20(4): 851-875.

Oden, J. T., J. M. Bass, C. Y. Huang, and C. W. Berry. 1990. "Recent Results on Smart Algorithms and Adaptive Methods for Two- and Three-Dimensional Problems in Computational Fluid Mechanics." Computers and Structures 35(4): 381-396.

Savant, G., C. J. Trahan, C. Berger, J. T, McAlpin, and T. O. McAlpin. 2018. "Refinement Indicator for DynamicMesh Adaption in Three-Dimensional Shallow-Water Equation Modeling," Journal of Hydraulic Engineering, ASCE 144(2). https://ascelibrary.org/doi/10.1061/\%28ASCE\%29HY.1943-7900.0001394

Sturm, T. W. 2001. Open Channel Hydraulics. New York: McGraw-Hill.

Tate, J. N., R. C. Berger, and R. L. Stockstill. 2006. "Refinement Indicator for Mesh Adaption in Shallow-Water Modeling." Journal of Hydraulic Engineering, ASCE 132(8): 847-857.

Thompson, J., Z. Warsi, and C. Mastin. 1985. Numerical Grid Generation: Foundations and Applications. New York: Elsevier. 
U.S. Army Engineer Research and Development Center (ERDC). 2019. Adaptive Hydraulics 2D Shallow Water (AdHSW2D) User's Manual (Version 4.6). Vicksburg, MS: U. S. Army Engineer Research and Development Center. Accessed June 29. https://chl.erdc.dren.mil/adh/documentation/AdH_Manual_Hydrodynamic-Version4.6.pdf

DISCLAIMER: The contents of this technical note are not to be used for advertising, publication, or promotional purposes. Citation of trade names does not constitute an official endorsement or approval of the use of such products. 